\title{
CORRIGENDUM
}

\section{High-yield self-limiting single-nanowire assembly with dielectrophoresis}

Erik M. Freer, Oleg Grachev, Xiangfeng Duan, Samuel Martin and David P. Stumbo

Nature Nanotechnology 5, 525-530 (2010); published online: 6 June 2010; corrected after print: 9 July 2010.

In the version of this Article originally published, Xiangfeng Duan and Samuel Martin were missing from the author list. Their names and affiliations have now been added to the HTML and PDF versions of the text. The supplementary information, acknowledgements, author contributions and competing financial interests statement have been amended. 